\title{
Functionalist Translation Theory and Its Application in Literary Translation
}

\author{
Dan Wang \\ College of Foreign Languages, Bohai University, Jinzhou, 121013, China \\ 1601698716@qq.com
}

Keywords: functionalist translation theory; literary translation; application; strategy

\begin{abstract}
Functionalist translation theory advocates the translator to analyze the original works. On the basis of clarifying the author's intention, the translator will translate different types of text and genre and translation purpose, combine the expectation of the target readers and the needs of translation initiators, provides an effective methodological guidance for the translation practice, to ensure the adequacy of the function of the target language in the target culture. According to literary translation ideas of the functionalist theory, combined with the characteristics and difficulties of literary translation, and puts forward some strategies of literary translation under the guidance of functional translation theories, including the understanding of the cultural background, text style, profound understanding of literary text, applying the functionalist theory schema method. The study results of this paper are an effective complement to the existing literary translation strategies, which are of great significance to the further study of functional translation theory and the improvement of the level of literary translation.
\end{abstract}

\section{Introduction}

The translation is based on accurate and fluent, one language into another language behavior. Literary works are a kind of art that are created by language, literary translation is to use another language tool to convey artistic conception of the original, so that readers can read the translation text as the original reading from the same as to be inspired to get touched and the feeling of the beauty. Translation is not a technical language variation, but the translator through the original language shape, deeply appreciate the original author of the process of artistic creation, and then in their own thoughts, feelings and life experience, find the most appropriate proof about the original content. At the same time, it is necessary to use the literary language suitable for the original style, and to reproduce the content and form of the original works without any doubt.

Compared with the fact that the importance of literary translation is increasing day by day, the importance of literary translation is decreasing. Literary translation is regarded as a technical work that anyone can do, as long as the text is literal and literally translated can be completed, regardless of the spirit purport of the word. This technical translation work, its degree of difficulty and importance, cannot be equal to the original literary creation. Literary works are the important ways to get through the cultural communication channels. Literary translation has become an important part of literary activities today.

New period of Chinese literature, every wave of ideas, and literary translation has a direct link. At present, there are many problems in literary translation. There is still a long distance between the current situation of literary translation and the trend of literary development and the needs of readers. The improvement of the level of the whole people does not bring the level of literary translation. On the one hand, the general level of literary translation does not reach the desired level, the high level of translation talent did not appear because of the increase of translation works; the other hand, foreign literary works translated into Chinese increasing at the same time, how to China literary works translated into the world has become a common problem. In this context, it is necessary to study literary translation. Based on the theory of functional translation, this paper offers new methods and approaches to improve the level of literary translation. 


\section{Development on Functionalist Translation Theory}

The theory of functional translation has been brought forward by the results of a series of research activities such as communicative theory, behavioral theory, discourse linguistics, discourse theory, and literary acceptance theory. It reflects the comprehensive turn of translation, that is, the dominant linguistics and the emphasis on the form, emphasizing the equivalence of translation theory, and turning to the more emphasis on functional and socio-cultural factors. The functionalist school argues that translation is an act. The theoretical emphasis is presented in the following three aspects: the interpretation of the substance of translation, the role analysis of the participants in the translation process, and the translation of functional principles. The development of functional translation theory is divided into three stages.

In the first stage, Rice classified the text function as a criterion of translation criticism, and proposed that "the special purpose to be achieved by the translation act" should be regarded as the new criterion of translation criticism. That is, to evaluate the translation from the relationship between the original and the translating functions. This relationship refers to the functional equivalence between the original text and the target text. Rice's text type classification, emphasizing different text should be taken for different translation strategies to achieve the text function. But its viewpoint still has the limitation. The theory is based on the equivalence theory, and thinks that the original and the translation function cannot be changed. In fact, the translation and the original text can have different functions.

In the second stage, with Fimmel's teleology as the symbol, Fermi 's teleology breaks through the restriction of equivalence theory and becomes one of the core theories of functional translation. Skopos theory holds that translation is a kind of cross-cultural communicative activity with clear purpose and intention, and under the influence of the translator, based on the original text. Translation is a kind of purposeful behavior, interpersonal behavior and communicative cultural behavior. In the past, translation theory holds that translation involves three aspects: the original author, the translator and the target receiver. The functionalist school also joined the sponsors of the translation process, the users and recipients and other participants.

In the third stage, Nord combines the norms of translation and function with the principles of loyalty into Vermeer's teleological framework. Mantali's translation behavior is regarded as a complex activity to achieve a specific purpose, and focuses on the roles of participants (initiators, translators, users of text, and recipients of information) and behavior of participants in the context conditions. Development enriched the teleology of Fermil, but also make up for the shortage of teleology. Functionalist theory gets rid of the shackles of equivalence theory. Translation is a cross-linguistic and cross-cultural activity, which emphasizes the subjective initiative of the translator and opens up a new way for the language school of western translation theorists.

\section{Opinion of Literary Translation on Functionalist Translation Theory}

Functional translation theory has always been regarded by some scholars as a theory that is more applicable to guide the translation of non-literary discourse, but some scholars have different views on whether the theory is applicable to literary translation. Such as Wenjun and Gao Xiaoying, explored the application of functionalist translation theory in literary translation criticism, and pointed out that "Functionalist translation theory, based on culture, broadens the perspective of translation, and the translation is extended to the dynamic behavior of the original author, the author integrated the readers, multiple relations category, thus providing a multi angle and dynamic concept for the standard of literary translation criticism.

Unlike the traditional equivalence theory, which emphasizes the study of lexical translation, functional translation theory raises translation studies to the level of discourse, changes the traditional word-based translation model, advocates cultural factors, takes translation as the first principle, and emphasizes target language context, considering the original author, translation sponsors, translators and readers of the multilateral relations, while emphasizing the cross-cultural communication function of translation. 


\section{Characteristics on Literary Translation}

The sound and rhythm, artistic conception and atmosphere of literary works, as well as the way of individual discourse determine the characteristics of literary translation. In order to maintain and reproduce the aesthetic value of literary works, the rhythm and sound of language, especially the rhythm of language, should be reflected in the translation. In order to obtain the same or similar aesthetic charm, literary translation must maintain and reproduce the atmosphere and artistic conception; The translator should study the author and express the language styles of different writers in another language.

Literary translation is subjective and creative. Therefore, the literary accomplishment and expressiveness of literary translation are the requirements of translators for literary translation. This is also the guarantee that the translator can fully understand the original works and fully express the original form, content, spiritual essence and artistic conception. At the same time, literary translation has the dependence and the attribute to the original works, so faithful to become the basic principles of literary translation. Literary translation is the unity of opposites between shape and spirit, in the process of literary translation, attention should be paid not only to the translation of the original language, but also to the author's emotional attitudes, original aesthetic thoughts and artistic conception. Literary translation is an important bridge between writers and readers. It bears the responsibility of inheriting the outstanding cultural treasures of the world. Therefore, literary translation requires the translator to have artistic awareness and proficiency in Language Arts.

\section{Difficulties on Literary Translation}

The difficulties of literary translation are reflected in the following four aspects:

(1) The problem of context transfer. In the process of translating literary works from one language into another, the first problem is the transfer of context. When translating literary works born in one cultural context into another cultural context, the translator is prone to image distortion and language deviation, which is caused by the anti-translation of context. For example, zodiac is a cultural symbol China unique, in the transformation in English and Chinese can only convey the basic surface meaning of zodiac, and its cultural meaning is difficult to convey.

(2) The perspective of consistency. In the course of literary translation, translation often does not conform to the Chinese logic, because the consistency of the perspective before and after the translation is neglected. In literary works, especially novels, the character perspective always runs through the article, played an extremely important role, which requires attention to consistency in the translation, to avoid confusion due to the perspective of the mistranslation.

(3) The transmission of cultural information. With the constant strengthening of communication among different countries, people have become more and more interested in foreign cultures, and people are no longer satisfied with the translation for the transfer of basic information, hoping to have a deeper and more thorough understanding of the cultural information of foreign cultures. This requires the translation of literary translation not only to translate the basic information of the original text, but also translated the original aesthetic information, which requires must pay attention to the translation of cultural images, to retain the image transmission or alternative way to better achieve cultural transmission.

(4) The reproduction of language style. Literature is the art of language. Literary language is the tool and medium of literature. Therefore, so the reproduction of the original style of language is a problem cannot be ignored in literary translation, which is a difficult literary translation, which requires translators to go deep into the original, constantly deepen the understanding and experience of original language features and language style, to achieve consistent and harmonious between translation and the original style.

\section{Literary Translation under the Guidance of Functionalist Translation Theory}

Faced with the special difficulties of literary translation, functional translation theory puts forward 
the following translation strategies: the author's intention can only be conveyed through the interpretation of the translator. Text is an open system, the specific form of the text is determined by the translator's understanding and translation purpose of the original; with reference to the sender's intention, the translator decided to complete what the function is, to take the literature translation to provide the reader with the full information from the content to the form, or just to obtain the similar function of the source text, take translation tools; The translated text should be processed according to the function of translation to decide whether it is to maintain the exotic flavor of the source text, to maintain the cultural distance, or adjust it to match the culture of the target language. The translator first analyzes the source text and then determines how to imitate the original text to complete the function in the target language environment, and achieve the effect of readers in the target language context. For example, it is a free translation to make the reader feel unable to read the translated text, or literal translation to enrich the expression of the target language. Combined with the results of previous studies, the strategy of this paper is as follows:

(1) Understanding of cultural background factors. Language, as an integral part of culture, is the carrier of culture, reflecting a nation's rich and colorful cultural phenomenon. Literature as a superstructure of society, it must reflect the social and cultural aspects. Therefore, literary translation must recognize and reflect the image of social life contained in the original works, or re-understand and reflect the social life in the original works. Without a deep understanding of the socio-cultural background of the source language, the translator cannot have a keen aesthetic and sociological vision, the understanding and re-creation of literary works cannot be carried out to a deeper level. Literature background factors include missionary beliefs, history and culture, life style and regional cultural differences.

(2) Grasp the style of the original text. Style is the artistic characteristic and creative individuality of writer and artist in creation. Because of writer's life experience, standpoint, artistic accomplishment and personality traits, they are different in handling subject matter, controlling genre and describing image. This style of writing and art is more important than the expression of meaning itself. Therefore, should understand the author's life, creative methods and background, and make comments and analysis; pay attention to the analysis of the text, including the tendency of words, creative methods, features of sentences and rhetorical devices.

(3) A deep understanding of the original literature. Literary translation requires the translator to have the writer's literary accomplishment and the expressive power of the artist, based in order to understand and grasp the essence of the original spirit of the original, the original content and form of artistic images all blend into one harmonious whole. Therefore, the translator vividly to translate literary works, a deep understanding of the works is the foundation. The translator only has his own deep understanding of the original, in order to use another language to re-create the works, in order to further convey the author's style and thinking. First, analyze and comprehend the linguistic components of the works, the literal and related meanings of the words, the structural and syntactic meaning and relevance, the literal meaning and relevance of the rhetorical devices; the second, to analyze and understand the literary elements of the works, including the background, plot, character, perspective, theme, structure and style.

(4) The schema method of functional translation theory. Schema is an important concept of cognitive psychology and psycholinguistics. It is the form of mental representations of knowledge. It is the general knowledge of the world stored in the mind and the basis of knowing and understanding the world. The essence of translation is the translation of schemata, that is, the various schemas in the source language are reproduced in the target language. According to the contents of the schema is divided into language schema, contextual schema and cultural schema. Language schemata play a complementary role, and contextual schemata play a decisive role in semantics. Cultural schemata help the reader to understand correctly. In the process of schema translation, the translator should correctly decode the linguistic schema, contextual schema and cultural schema in the source language, and re-encode the target language just perfect, in order to make the reader fully understand the information contained in the source language. 


\section{Conclusion}

Literary translation is a process of communication and communication tool between two linguistic societies in the field of literature. The aim is to promote the political, economic and cultural progress of this linguistic society. The task is to integrate a certain social life image contained in the original, completely from one language to another language, so that the reader can read the translation as the original can be the same as the inspiration, touch and beauty to enjoy. Translation is a debate between science and art, but literary translation is always an art, but also an advanced form of art. Functional translation theory, from the perspective of the translation' purpose, avoids the dilemma of domestication and foreignization. The translator of functional translation theory, Nord put forward the principles of loyalty to solve the changeable relationship between the translator and the original author and the target readers. The functional translation theory of multiple criteria based on the Skopos theory as the criterion, puts forward a feasible translation program, making the literary translation is more objective, more normative and evidence-based, a good guide for literary translation. The translation strategy proposed in this paper is an effective complement to the existing literary translation strategies, which is of great significance for deepening functional translation studies and improving the level of literary translation.

\section{References}

[1] H. M. Su, "On Functionalist Translation Theory and Literary Translation," Journal of Changchun Normal University (Humanities and Social Sciences), vol. 25, no. 4, pp. 121-124, 2006.

[2] Q. Jie, "Reflection on Literary Dialect Translation in Light of Functional Translation Theory," Master's degree of Agricultural University of Hebei, 2013.

[3] T. Li, "An analysis of English translation skills from the perspective of functional translation theory," English Square(Academic Research), vol. 3, no. 2, pp. 19-19, 2013.

[4] Skyline community, "What is the function of translation theory," http://wenda.tianya.cn/question/738f29cccfdfd037, 2011-1-6.

[5] H. Y. Cheng, "A functional critique of the methods of literary translation," Journal of Pingdingshan University, vol. 20, no. 3, pp. 73-75, 2005.

[6] Q. Y. Huang, "A study of business English translation based on functional translation theory," Contemporary Education Research and Teaching Practice, vol. 3, no. 8, pp. 99-100, 2015. 